\title{
Constraint Management in Li-ion Batteries: A Modified Reference Governor Approach
}

\author{
S. J. Moura, N. A. Chaturvedi, and M. Krstić
}

\begin{abstract}
This paper addresses the problem of satisfying state constraints in Li-ion batteries, to maintain safe operation and prolong battery life. Mathematically, these constraints are formulated from a first principles electrochemical model. Consequently, the constraints explicitly model specific degradation mechanisms, such as lithium plating, lithium depletion, overheating, and stress fracture. The critical challenges, however, are that (i) these states evolve according to a system of nonlinear partial differential equations, and (ii) the states are not physically measurable. This paper focuses on the first challenge by utilizing the reference governor concept. The results demonstrate how electrochemical model state information can be utilized to ensure safe operation, while providing opportunities to enhance energy capacity, power, and charge times in Li-ion batteries.
\end{abstract}

\section{INTRODUCTION}

This paper develops a reference governor-based approach to operating lithium-ion batteries at their safe operating limits.

Battery energy storage is a key enabling technology for portable electronics, electrified transportation, renewable energy integration, and smart grids. A crucial obstacle to the proliferation of battery energy storage is cost. Specifically, battery packs are typically oversized and underutilized to ensure longevity and robust operation. Indeed, oversizing mitigates several degradation mechanisms, such as lithiumplating, lithium depletion/over-saturation, overheating, and stress fractures by reducing C-rates. However, oversizing can be overly conservative. This paper seeks to eliminate this conservatism by developing reference governor-based tools that enable smaller-sized batteries whose states satisfy operating constraints that explicitly model degradation mechanisms. This is in contrast to the traditional approach, which utilizes voltage and current constraints that do not necessarily correspond to the internal degradation mechanisms.

A reference governor (RG) is an effective tool for controlling a system within pointwise-in-time constraints. This add-on device attenuates the command signal (electric current, in our case) such that state constraints are satisfied while maintaining tracking performance [1], [2]. This method has been applied to a variety of systems, including electrochemical energy conversation devices. For example, Sun and Kolmanovsky developed a robust nonlinear RG to protect

This work was supported by the University of California Presidential Postdoctoral Fellowship Program and the National Science Foundation.

S. J. Moura and M. Krstic are with the Department of Mechanical and Aerospace Engineering and Cymer Center for Control Systems and Dynamics at the University of California, San Diego, CA 92093-0411, USA (e-mail: smoura@ucsd.edu; krsticeucsd.edu)

N. A. Chaturvedi is with Robert Bosch LLC, Research \& Technology Center, Palo Alto, CA 94304 USA (e-mail: nalin. chaturvedi@us.bosch.com)

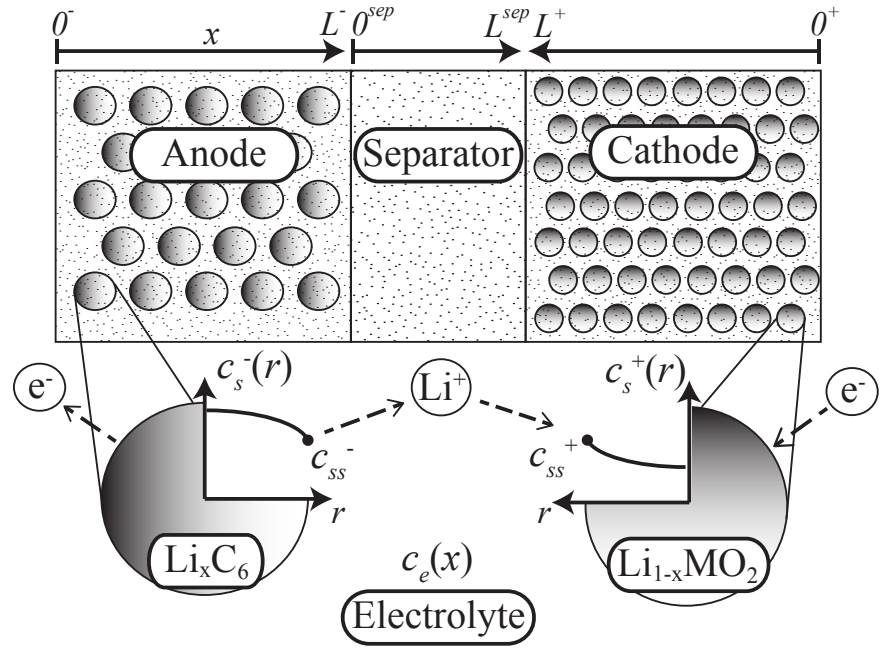

Fig. 1. Schematic of the Doyle-Fuller-Newman model [8]. The model considers two phases: the solid and electrolyte. In the solid, states evolve in the $x$ and $r$ dimensions. In the electrolyte, states evolve in the $x$ dimension only. The cell is divided into three regions: anode, separator, and cathode.

against oxygen starvation in fuel cell systems [3]. In [4], Vahidi et al. adopted a so-called "Fast" RG approach for fuel cells to protect against compressor surge/chock and oxygen starvation. In battery systems, Plett designed an algorithm to determine power limits in real-time [5]. This approach considers an equivalent circuit model and terminal voltage constraints. Smith et al. recently utilized a reduced-order, linearized electrochemical model for state estimation and prediction of maximum, safe current draw [6]. Klein et al. use detailed electrochemical model with nonlinear model predictive control to determine optimal charging trajectories, in the presence of state constraints [7].

In this paper, we seek to design a scheme which governs a commanded electrical current, in the presence of constraints on the electrochemical states. As such, this paper provides two important designs for constraint management in batteries: (i) a nonlinear modified RG which utilizes an electrochemical model, and (ii) a linear modified RG which utilizes a linearized electrochemical model. These algorithms enable us to ensure safe operation, while providing possibilities for enhancing performance.

The remainder of this paper is structured as follows. Section II summarizes the electrochemical model and presents two motivating examples. Sections III and IV develop the nonlinear and linearized modified RGs, respectively. Conclusions are summarized in Section V. 


\section{ELECTROCHEMicAl Model \& Motivating ExAMPLES}

\section{A. Doyle-Fuller-Newman Model}

We consider the Doyle-Fuller-Newman (DFN) model in Fig. 1 to predict the evolution of lithium concentration in the solid $c_{s}^{ \pm}(x, r, t)$, lithium concentration in the electrolyte $c_{e}(x, t)$, solid electric potential $\phi_{s}^{ \pm}(x, t)$, electrolyte electric potential $\phi_{e}(x, t)$, ionic current $i_{e}^{ \pm}(x, t)$, molar ion fluxes $j_{n}^{ \pm}(x, t)$, and bulk cell temperature $T(t)$ [8]. The governing equations are given by

$$
\begin{aligned}
\frac{\partial c_{s}^{ \pm}}{\partial t}(x, r, t) & =\frac{1}{r^{2}} \frac{\partial}{\partial r}\left[D_{s}^{ \pm} r^{2} \frac{\partial c_{s}^{ \pm}}{\partial r}(x, r, t)\right] \\
\varepsilon_{e} \frac{\partial c_{e}}{\partial t}(x, t) & =\frac{\partial}{\partial x}\left[\varepsilon_{e} D_{e} \frac{\partial c_{e}}{\partial x}(x, t)+\frac{1-t_{c}^{0}}{F} i_{e}^{ \pm}(x, t)\right] \\
\frac{\partial \phi_{s}^{ \pm}}{\partial x}(x, t) & =\frac{i_{e}^{ \pm}(x, t)-I(t)}{\sigma^{ \pm}} \\
\frac{\partial \phi_{e}}{\partial x}(x, t) & =-\frac{i_{e}^{ \pm}(x, t)}{\kappa}+\frac{2 R T}{F}\left(1-t_{c}^{0}\right) \\
& \times\left(1+\frac{d \ln f_{c / a}}{d \ln c_{e}}(x, t)\right) \frac{\partial \ln c_{e}}{\partial x}(x, t) \\
\frac{\partial i_{e}^{ \pm}}{\partial x}(x, t) & =a_{s} F j_{n}^{ \pm}(x, t) \\
j_{n}^{ \pm}(x, t) & =\frac{1}{F} i_{0}^{ \pm}(x, t)\left[e^{\frac{\alpha_{a} F}{R T} \eta^{ \pm}(x, t)}-e^{-\frac{\alpha_{c} F}{R T} \eta^{ \pm}(x, t)}\right] \\
\rho^{\mathrm{avg}} c_{P} \frac{d T}{d t}(t) & =h_{\mathrm{cell}}\left[T_{\mathrm{amb}}(t)-T(t)\right]+I(t) V(t) \\
& -\int_{0^{-}}^{0^{+}} a_{s} F j_{n}(x, t) \Delta T(x, t) d x
\end{aligned}
$$

where $D_{e}, \kappa, f_{c / a}$ are functions of $c_{e}(x, t)$ and

$$
\begin{aligned}
i_{0}^{ \pm}(x, t)= & k^{ \pm}\left[c_{s s}^{ \pm}(x, t)\right]^{\alpha_{c}}\left[c_{e}(x, t)\left(c_{s, \max }^{ \pm}-c_{s s}^{ \pm}(x, t)\right)\right]^{\alpha_{a}} \\
\eta^{ \pm}(x, t)= & \phi_{s}^{ \pm}(x, t)-\phi_{e}(x, t) \\
& -U^{ \pm}\left(c_{s s}^{ \pm}(x, t)\right)-F R_{f}^{ \pm} j_{n}^{ \pm}(x, t) \\
c_{s s}^{ \pm}(x, t)= & c_{s}^{ \pm}\left(x, R_{s}^{ \pm}, t\right) \\
\Delta T(x, t)= & U^{ \pm}\left(\bar{c}_{s}^{ \pm}(x, t)\right)-T(t) \frac{\partial U^{ \pm}}{\partial T}\left(\bar{c}_{s}^{ \pm}(x, t)\right) \\
\bar{c}_{s}^{ \pm}(x, t)= & \frac{3}{\left(R_{s}^{ \pm}\right)^{3}} \int_{0}^{R_{s}^{ \pm}} r^{2} c_{s}^{ \pm}(x, r, t) d r
\end{aligned}
$$

Along with these equations are corresponding boundary and initial conditions. For brevity, we only summarize the equations here. However, further details, including notation definitions, can be found in [8], [9]. The parameters are taken from the publicly available DUALFOIL model, developed by Newman and his collaborators [10]. The simulations provided here correspond to a $\mathrm{LiCoO}_{2}$ cell. However, the techniques are broadly applicable to any Li-ion chemistry.

\section{B. Constraints}

During operation it is critical to maintain the battery within a safe operating regime. This protects the battery against failure and maintains longevity. Towards this end, we consider several constraints,

$$
\begin{aligned}
I_{\min } & \leq I(t) \leq I_{\max }, \\
\theta_{\min }^{ \pm} & \leq \frac{c_{s}^{ \pm}(x, r, t)}{c_{s, \max }^{ \pm}} \leq \theta_{\max }^{ \pm}, \\
c_{s, r, \min }^{ \pm} & \leq \frac{\partial c_{s}^{ \pm}}{\partial r}(x, r, t) \leq c_{s, r, \max }^{ \pm}, \\
c_{e, \min } & \leq c_{e}(x, t) \leq c_{e, \max }, \\
T_{\min } & \leq T(t) \leq T_{\max }, \\
\eta_{s}(x, t) & =\phi_{s}(x, t)-\phi_{e}(x, t)-U_{s} \geq 0 .
\end{aligned}
$$

Equation (13) signifies that the power electronics provide finite current. Equation (14) protects the solid active material from lithium depletion or over-saturation. Equation (15) constrains mechanical stress induced by spatial concentration gradients, which is known to cause particle fracture [11]. Equation (16) protects the electrolyte from lithium depletion or over-saturation. Equation (17) protects against excessively cold or hot temperatures, which accelerates cell aging. Finally, Eqn. (18) is a side reaction overpotential constraint. This constraint avoids regimes where unwanted side reactions occur, including lithium plating and solid/electrolyte interphase film formation [9].

\section{Numerical Implementation}

Numerical solution of the coupled nonlinear PDAE (1)-(12) is, by itself, a nontrivial task. In fact, a body of literature exists on this singular topic (see, e.g. [12]-[14]). In our work the PDEs governing Fickian diffusion in the solid phase, (1), are discretized in the $r$-dimension via Padé approximates [14]. All the remaining PDEs are discretized in the $x$ dimension via the central difference method [15], such that the mass of lithium is conserved. This ultimately produces a finitedimensional continuous-time differential-algebraic equation (DAE) system

$$
\begin{aligned}
\dot{x}(t) & =f(x(t), z(t), I(t)), \\
0 & =g(x(t), z(t), I(t)),
\end{aligned}
$$

where

$$
x=\left[c_{s}^{ \pm}, c_{e}, T\right]^{T}, \quad z=\left[\phi_{s}^{ \pm}, i_{e}^{ \pm}, \phi_{e}, j_{n}^{ \pm}\right]^{T} .
$$

This DAE model is then propagated forward in time via an implicit numerical scheme. In particular, the nonlinear discretized equations are solved via Newton's method, at each time step. A crucial step is to provide the scheme with analytic expressions for the Jacobian, which ensures fast convergence and accurate simulations. These Jacobians are also used for the linearized modified reference governor design in Section IV.

\section{Motivating Examples}

Next, we consider two motivating examples: lithium plating and lithium depletion in the electrolyte. In Fig. 2 we consider a $10 \mathrm{sec}, 3 \mathrm{C}$ pulse charging cycle at $90 \%$ SOC as an example scenario when lithium plating may occur. In addition to the voltage response, Fig. 2 displays the 

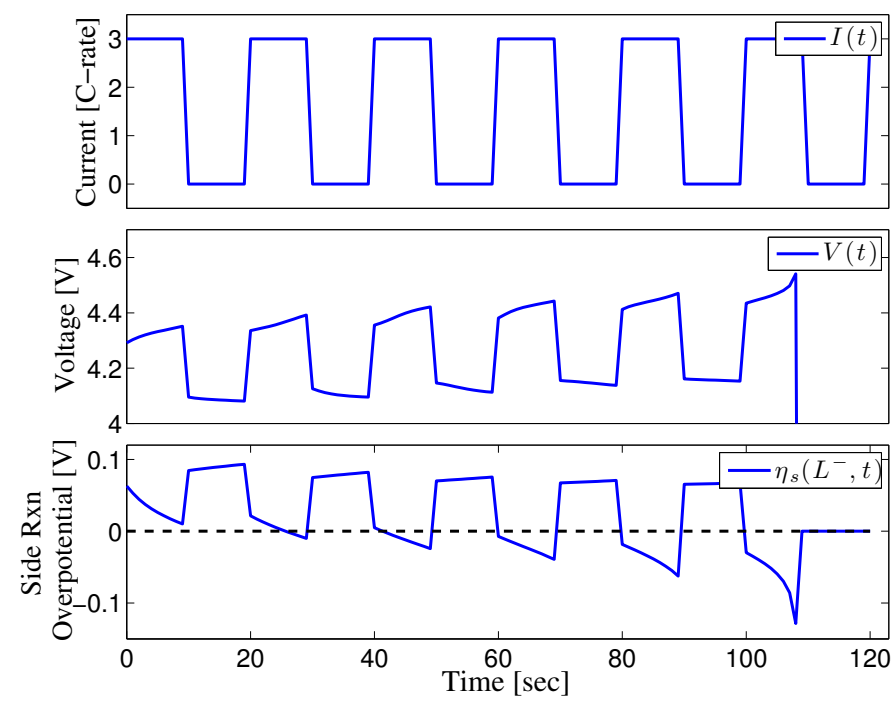

Fig. 2. Motivating example of Li plating during $10 \mathrm{sec} 3 \mathrm{C}$ pulse charging.
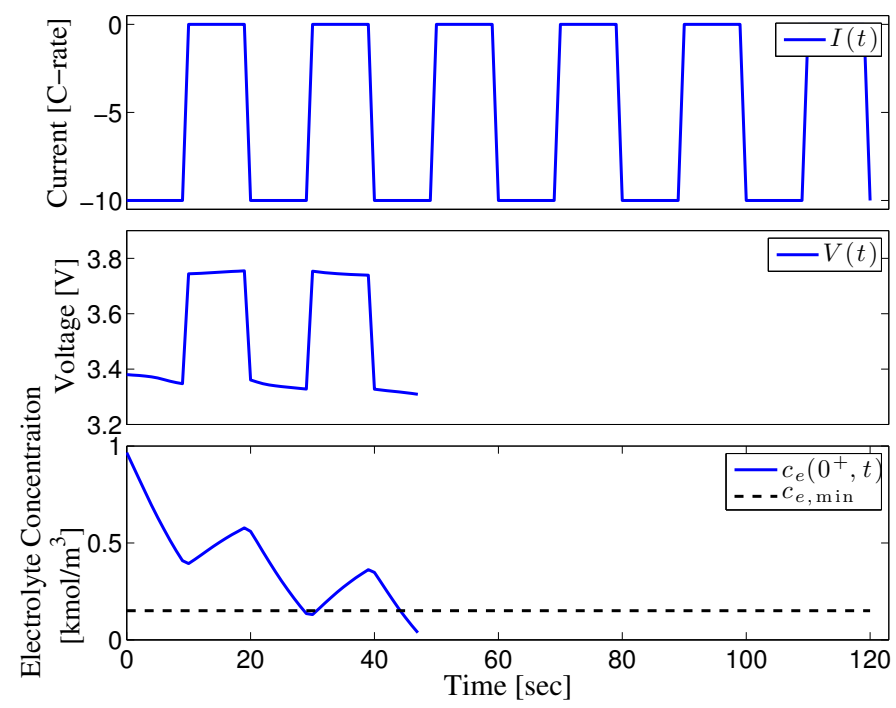

Fig. 3. Motivating example of lithium depletion in the electrolyte during $10 \mathrm{sec} 10 \mathrm{C}$ pulse discharging. The model is invalid after $c_{e}\left(0^{+}, t\right)<0$.

side reaction overpotential response at the anode/separator interface, $\eta_{s}\left(L^{-}, t\right)$. Note that $\eta_{s}\left(L^{-}, t\right)<0$ over several time periods. This induces lithium plating, leading to dendrite formation that may potentially short-circuit the electrodes.

Figure 3 displays responses for $10 \mathrm{sec}, 10 \mathrm{C}$ pulse discharging cycle at $60 \%$ SOC. Under this scenario, Li is eventually depleted at the cathode/current collector interface, denoted by $c_{e}\left(0^{+}, t\right)$. The model becomes invalid when $c_{e}\left(0^{+}, t\right)<0$.

In the following sections, we design an algorithm to protect the battery from entering these unsafe regions.

\section{Modified RefEREnCE Governor (MRG)}

\section{A. MRG Design}

We utilize the reference governor concept to handle constraint satisfaction in batteries. A reference governor is an add-on system that guarantees constraint satisfaction and maintains a desired level of reference tracking. It operates in

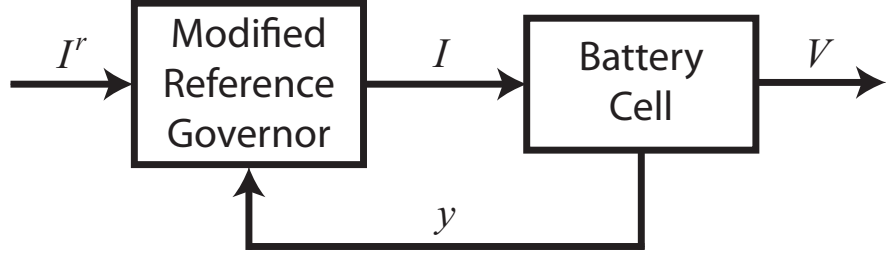

Fig. 4. Block diagram of modified reference governor with direct measurements of the constrained variables $y$.

a discrete-time domain, since the computations may not be feasibly performed in real-time. In our "modified" reference governor approach, the applied current $I(t)$ and reference current $I^{r}(t)$ are related according to

$$
I[k+1]=\beta[k] I^{r}[k], \quad \beta \in[0,1],
$$

where $I(t)=I[k]$ for $t \in[k \Delta t,(k+1) \Delta t), k \in \mathbb{Z}$, and similarly for $I^{r}[k]$. We define the admissible set

$$
\mathcal{O}=\left\{(x(t), z(t)): y(\tau) \in \mathcal{Y}, \forall \tau \in\left[t, t+T_{s}\right]\right\},
$$

where

$$
\begin{aligned}
\dot{x}(t) & =f\left(x(t), z(t), \beta I^{r}\right), \\
0 & =g\left(x(t), z(t), \beta I^{r}\right), \\
y(t) & =C_{1} x(t)+C_{2} z(t)+D \cdot \beta I^{r}+E .
\end{aligned}
$$

The output variables $y=\left[I, c_{s}^{ \pm}, c_{e}, T, \eta_{s}\right]^{T}$ must exist in set $\mathcal{Y}$, characterized by inequalities (13)-(18). The goal is to find the maximum value of $\beta$ which maintains the state in $\mathcal{O}$

$$
\beta^{*}[k]=\max \{\beta \in[0,1]:(x(t), z(t)) \in \mathcal{O}\},
$$

where $(x(t), z(t))$ depends on $\beta$ via (23)-(26).

To determine parameter $\beta^{*}$ at each time instant, the model is simulated forward over the time interval $\left[t, t+T_{s}\right]$, where $T_{s}$ is the simulation horizon. If the constraints are violated for a given value of $\beta$, then $\beta$ is reduced and the model is re-simulated to ascertain constraint satisfaction of the new value of $\beta$. If the constraints are satisfied, then $\beta$ is increased to reduce tracking error between $I(t)$ and $I^{r}(t)$. This process is iterated according to the bisection algorithm [15].

Remark 1: We refer to (22) as a "modified" reference governor to distinguish it from the conventional reference governor concept

$$
I[k+1]=I[k]+\beta[k]\left(I^{r}[k]-I[k]\right), \quad \beta \in[0,1],
$$

which inserts a low-pass filter between the reference and applied inputs [1], [2]. In the battery application, we find that (22) provides less conservative and more robust performance than (28), since it directly modifies the current as opposed to filtering it. A similar concept is used for batteries in [6].

\section{B. Simulations}

We consider the case when the constrained output variables, $y$, are measurable, as shown in Fig. 4. In practice, one needs to estimate these variables from measurements of current and voltage, as done in [16]. This section analyzes performance under the hypothetical situation of output variable feedback. 

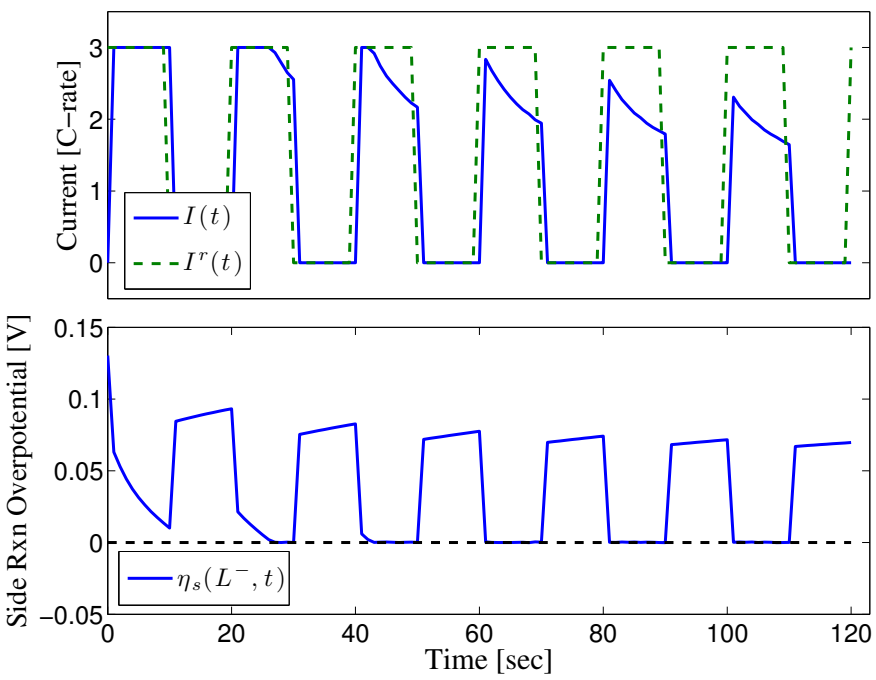

Fig. 5. Evolution of current $I(t)$, reference current $I^{r}(t)$, and side reaction overpotential $\eta_{s}\left(L^{-}, t\right)$ for a $10 \sec 3 \mathrm{C}$ pulse charging scenario, with a modified reference governor.
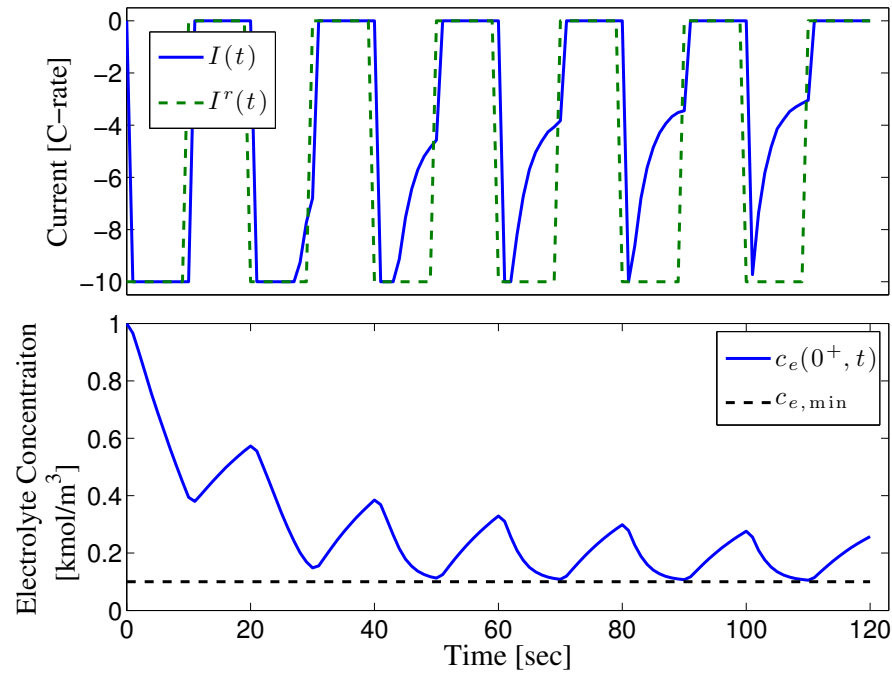

Fig. 6. Evolution of current $I(t)$, reference current $I^{r}(t)$, and electrolyte concentration $c_{e}\left(0^{+}, t\right)$ for a $10 \mathrm{sec} 8 \mathrm{C}$ pulse discharging scenario, with a modified reference governor.

In the following, we apply the MRG to the scenarios described in Section II-D. Figure 5 displays the current $I(t)$, reference current $I^{r}(t)$, and side reaction overpotential $\eta_{s}\left(L^{-}, t\right)$ for a $10 \mathrm{sec} 3 \mathrm{C}$ pulse charging scenario. Note how the MRG attenuates the current to satisfy $\eta_{s}>0$. Similarly, Fig. 6 displays the system responses for a $10 \mathrm{sec} 8 \mathrm{C}$ pulse discharging scenario. Again, $I(t)$ is attenuated such that lithium is not depleted in the electrolyte.

Next we demonstrate the benefits of utilizing a MRG for charging. Figure 7 compares the standard charging protocol, constant charging-constant voltage (CCCV), to a reference governor-based charging. In both cases, we consider a constant $1 \mathrm{C}$ charging current. The $\mathrm{CCCV}$ protocol applies 1C charging until the terminal voltage reaches a "maximum

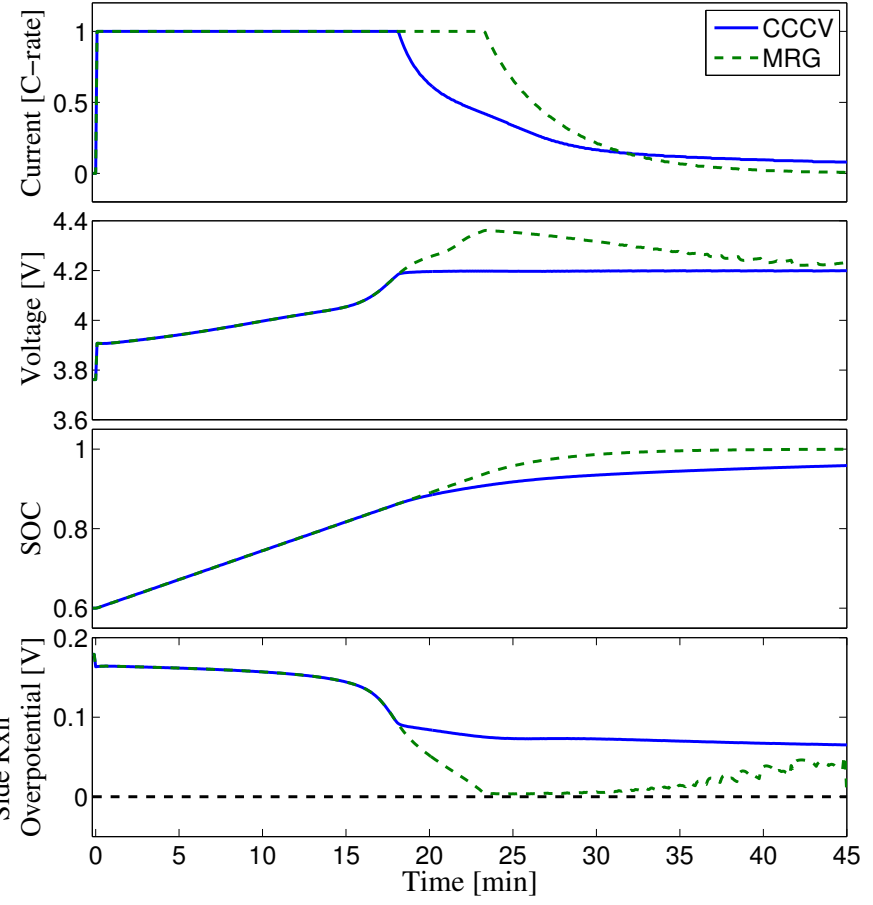

Fig. 7. Comparison of CCCV and modified reference governor (MRG) charging. The MRG regulates $\eta_{s}$ near its limit, thereby achieving $95 \%$ SOC in $24 \mathrm{~min}$ vs. $38 \mathrm{~min}$ for $\mathrm{CCCV}$, despite voltage exceeding $4.2 \mathrm{~V}$.

safe voltage level," $4.2 \mathrm{~V}$ in this case. This occurs near the 18 min mark. At this point, CCCV regulates terminal voltage at the maximum value, $4.2 \mathrm{~V}$, while the current diminishes toward zero. The maximum value of $4.2 \mathrm{~V}$ is selected such that lithium plating does not occur due to overcharging. Indeed, the side reaction overpotential does not fall below zero. However, this approach is conservative. Specifically, the side reaction overpotential can be regulated closer to its limit. The MRG applies $1 \mathrm{C}$ charging subject to the constraint $\eta_{s}\left(L^{-}, t\right) \geq 0$. In Fig. 7 the MRG maintains $\eta_{s} \geq 0$ despite voltage exceeding $4.2 \mathrm{~V}$. Moreover, the cell attains $95 \%$ SOC in $24 \mathrm{~min}$ vs $38 \mathrm{~min}$ for CCCV. Also note that CCCV reaches an equilibrium SOC of $96 \%$, whereas the RG achieves $100 \%$ SOC. Consequently, charging time is decreased by $37 \%$ and energy capacity is increased by $4 \%$.

\section{Linear Modified Reference Governor}

The nonlinear MRG developed in the previous section achieves guaranteed constraint satisfaction at the expense of computational effort. Computational complexity, however, is often the deciding factor on which design ultimately reaches implementation. Next we design and evaluate a computationally efficient MRG based upon a linearized model. The critical benefit of the linear MRG is that the parameter $\beta$ can be determined by an explicit expression. In contrast, the nonlinear MRG requires simulations and optimization.

\section{A. Linear MRG Design}

At each time step we linearize the model around the state and input values from the previous time step: $\left(x^{0}, z^{0}, u^{0}\right)=$ 


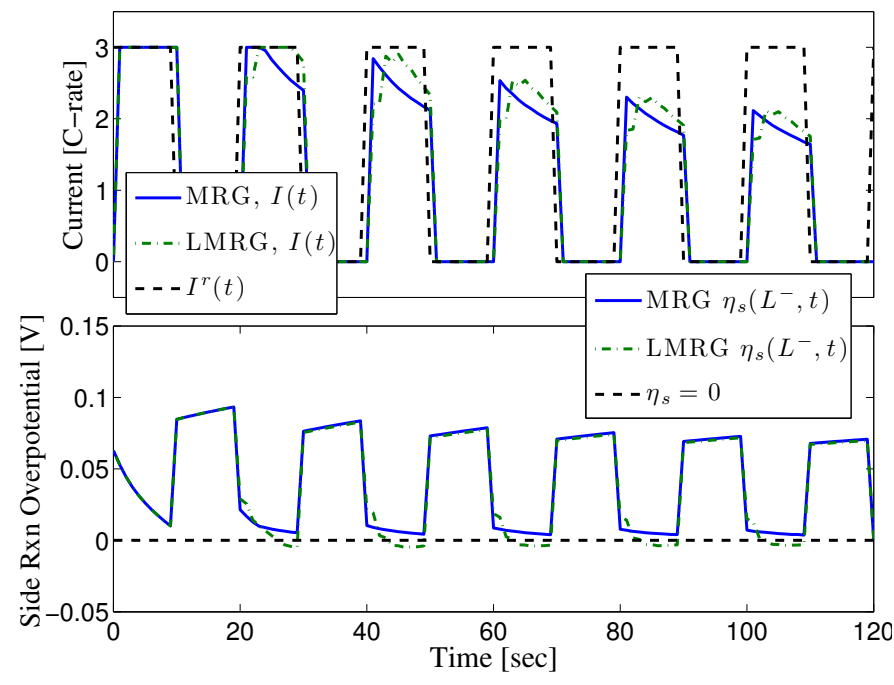

Fig. 8. Comparison of modified reference governor (MRG) and linear modified reference governor (LMRG). The signals depict the evolution of current $I(t)$, reference current $I^{r}(t)$, and side reaction overpotential $\eta_{s}\left(L^{-}, t\right)$ for a $10 \sec 3 \mathrm{C}$ pulse charging scenario. Note that the LMRG violates the constraint, due to modeling errors induced by linearization.

$(x[k-1], z[k-1], I[k-1])$ to obtain evolution equations

$$
\begin{aligned}
& \dot{\tilde{x}}=A_{11} \tilde{x}+A_{12} \tilde{z}+B_{1} \tilde{I}, \\
& 0=A_{21} \tilde{x}+A_{22} \tilde{z}+B_{2} \tilde{I},
\end{aligned}
$$

where $\tilde{x}=x-x^{0}, \tilde{z}=z-z^{0}, \tilde{I}=\beta I^{r}-I^{0}$ and $A_{11}, A_{12}, A_{21}, A_{22}, B_{1}, B_{2}$ are the Jacobian terms of the nonlinear state equations (24)-(25), evaluated at $\left(x^{0}, z^{0}, u^{0}\right)$. Since this DAE system is linear, we can explicitly solve for $\tilde{z}$ and write the system as

$$
\dot{\tilde{x}}=A \tilde{x}+B \tilde{I}
$$

where $A=A_{11}-A_{12} A_{22}^{-1} A_{21}$ and $B=B_{1}-A_{12} A_{22}^{-1} B_{2}$. Under this representation, the states after a simulation horizon horizon of $T_{s}$, can be computed explicitly. That is,

$$
\begin{aligned}
& \tilde{x}\left(t+T_{s}\right)=e^{A T_{s}} \tilde{x}(t)+\int_{t}^{t+T_{s}} e^{A\left(t+T_{s}-\tau\right)} B \tilde{I} d \tau \\
& \tilde{z}\left(t+T_{s}\right)=-A_{22}^{-1}\left[A_{21} \tilde{x}\left(t+T_{s}\right)+B_{2} \tilde{I}\right] .
\end{aligned}
$$

The constrained output variables after $T_{s}$ time units are then given by

$$
\begin{aligned}
y\left(t+T_{s}\right)= & C_{1}\left[x^{0}+\tilde{x}\left(t+T_{s}\right)\right]+C_{2}\left[z^{0}+\tilde{z}\left(t+T_{s}\right)\right] \\
& +D \cdot \beta I^{r}+E \leq 0
\end{aligned}
$$

where $C_{1}, C_{2}, D, E$ are matrices which incorporate inequalities (13)-(18). We also assume the reference current $I^{r}$ is constant over the simulation horizon - a typical assumption in RG design [1]-[4], [6]. We are now positioned to formulate the linearized MRG problem. Given the current states and reference current $\left(x(t), z(t), I^{r}(t)\right)$, solve

$$
\begin{aligned}
\max _{\beta \in[0,1]} & \beta \\
\text { subject to } & \beta F \leq G
\end{aligned}
$$
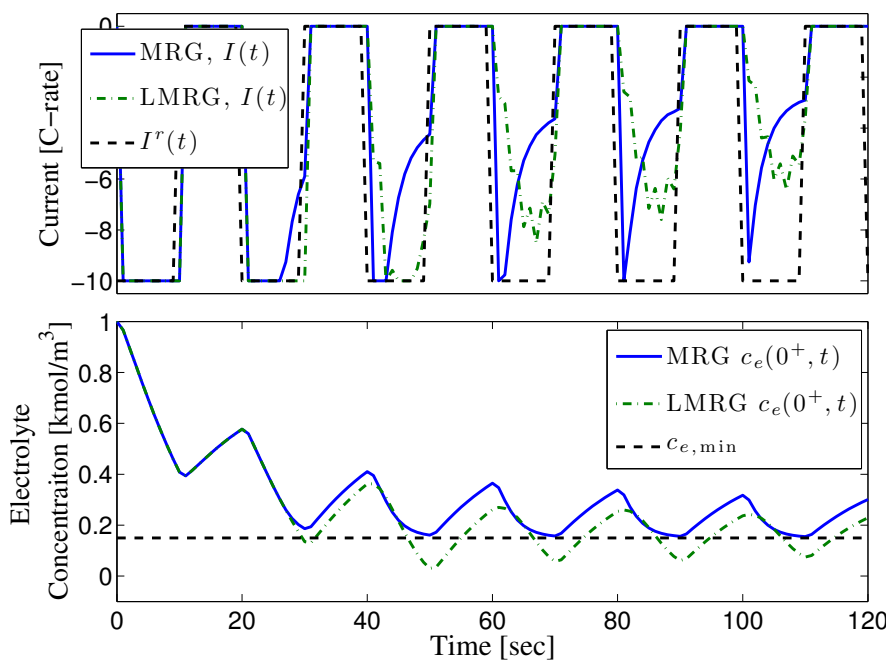

Fig. 9. Comparison of modified reference governor (MRG) and linear modified reference governor (LMRG). The signals depict the evolution of current $I(t)$, reference current $I^{r}(t)$, and electrolyte concentration $c_{e}\left(0^{+}, t\right)$ for a $10 \mathrm{sec} 10 \mathrm{C}$ pulse discharging scenario. Note that the LMRG violates the constraint, due to modeling errors induced by linearization.

where $F, G$ are vectors that incorporate the constraints (13)(18) and depend on $x(t)$ and $I^{r}(t)$ as follows

$$
\begin{aligned}
F= & {\left[C_{1} L-C_{2} A_{22}^{-1}\left(A_{21} L+B_{2}\right)+D\right] I^{r}, } \\
G= & -E-C_{1}\left[x^{0}+\Phi\left(x(t)-x^{0}\right)-L I^{0}\right] \\
& -C_{2}\left[z^{0}-A_{22}^{-1}\left[A_{21}\left(\Phi\left(x(t)-x^{0}\right)-B_{2} I^{0}\right]\right],\right.
\end{aligned}
$$

where

$$
\Phi=e^{A T_{s}}, \quad L=\int_{t}^{t+T_{s}} e^{A\left(t+T_{s}-\tau\right)} B d \tau .
$$

The optimization problem (35)-(36) is a one-dimensional linear program. Consequently, it can be solved explicitly as

$$
\begin{aligned}
& H_{i}=\left\{\begin{array}{ll}
G_{i} / F_{i} & \text { if } \quad F_{i}>0 \\
-G_{i} / F_{i} & \text { else }
\end{array} \quad i=1,2, \ldots, N_{c},\right. \\
& \beta^{*}=\min \left\{1, H_{i} \quad \mid i=1,2, \ldots, N_{c}\right\},
\end{aligned}
$$

where $G_{i}$ and $F_{i}$ denote the $i^{\text {th }}$ element of $G$ and $F$, respectively, and $N_{c}$ is the total number of elements.

\section{B. Simulations}

Next we evaluate simulations of the linear MRG to ascertain the trade off between computational efficiency and constraint satisfaction.

Figure 8 compares the linear modified reference governor (LMRG) to the nonlinear MRG, for the $10 \mathrm{sec} 3 \mathrm{C}$ pulse charging scenario. Note that in the $\mathrm{LMRG}, \eta_{s}\left(L^{-}, t\right)$ violates the constraint over several time periods, due to modeling errors induced by linearization. In practice, one could apply a more conservative constraint to offset any potential constraint violation. A similar result is portrayed in Fig. 9, for the $10 \mathrm{sec}$ 10C pulse discharging scenario, where $c_{e}\left(0^{+}, t\right)$ violates the constraint over several time periods. Nonetheless, the magnitude of constraint violation is relatively small and the 
TABLE I

COMPARISON OF CPU TIME FOR NONLINEAR AND LINEAR MRGS.

\begin{tabular}{c|c|c}
\hline \hline Scenario & MRG & Linear MRG \\
\hline 10sec 3C charging & $4.27 \mathrm{~min}(100 \%)$ & $1.03 \mathrm{~min}(24 \%)$ \\
10sec 10C discharging & $4.99 \mathrm{~min}(100 \%)$ & $1.13 \mathrm{~min}(23 \%)$ \\
\hline \hline
\end{tabular}

LMRG would be effective at mitigating degradation and prolonging battery life.

The critical advantage of the LMRG, however, is the increased computational efficiency. That is, the LMRG computes $\beta$ via the explicit expressions (40)-(42), whereas the nonlinear MRG requires nonlinear simulations and optimization. We consider the CPU time for each MRG as one indication of computational efficiency. The data provided in Table I indicates that the linear MRG reduces CPU time by over four-fold on a $2.7 \mathrm{GHz}$ dual-core laptop with 4GB of RAM. Further improvements are possible via code optimization.

Remark 2 (Current Limits \& Power Capacity): The linear MRG also provides real-time estimates of the maximum safe current and power capacity. The limiting current is given by

$$
I_{\lim }(t)=I^{r}(t) \cdot \min \left\{H_{i} \quad \mid \quad i=1,2, \ldots, N_{c}\right\},
$$

and the corresponding instantaneous power capacity is

$$
P_{\text {cap }}(t)=I_{\text {lim }}(t) V(t) .
$$

These variables are useful for feedback to higher-level supervisory control systems [5], [6], [17], [18].

\section{CONCLUSION}

This paper develops a reference governor-based approach to satisfying state constraints in batteries. Ultimately, this approach maintains the battery within a safe operational envelope, as defined by the electrochemical states. In addition, it enables one to enhance power capacity, energy capacity, and charging speed by eliminating the conservatism imposed by traditional operating constraints (e.g. voltage and current limits). The key ingredients to this approach are the following. First, we utilize a first principles electrochemical model to predict and constrain the evolution of physical degradation mechanisms. Second, a nonlinear modified reference governor (MRG) algorithm is developed assuming measurements of the constrained variables. Third, a linearized MRG is developed, which replaces simulations with an explicit function evaluation at the expense of possible constraint dissatisfaction.

In this paper we have assumed full state measurements and known parameters. Future work will combine the MRGs developed here with state and parameter estimates generated by the adaptive PDE observer developed in [16]. This outputfeedback system will (i) guard against harmful operating regimes, (ii) increase energy capacity, power capacity, and charging speed, and (iii) monitor state-of-charge and stateof-health, all from measurements of voltage, current, and temperature. Finally, we plan to quantify the aforementioned benefits by comparing these electrochemical-based algorithms against traditional battery management systems, on plug-in hybrid electric vehicle-like charge/discharge cycles.

\section{REFERENCES}

[1] E. Gilbert, I. Kolmanovsky, and K. Tan, Discrete-time reference governors and the nonlinear control of systems with state and control constraints. Wiley Online Library, 1995, vol. 5, no. 5.

[2] A. Bemporad, "Reference governor for constrained nonlinear systems," IEEE Transactions on Automatic Control, vol. 43, no. 3, pp. 415-419, 1998.

[3] J. Sun and I. V. Kolmanovsky, "Load governor for fuel cell oxygen starvation protection: A robust nonlinear reference governor approach," IEEE Transactions on Control Systems Technology, vol. 13, no. 6, pp. $911-920,2005$.

[4] A. Vahidi, I. Kolmanovsky, and A. Stefanopoulou, "Constraint handling in a fuel cell system: A fast reference governor approach," IEEE Transaction on Control Systems Technology, vol. 15, no. 1, pp. 86-98, 2007.

[5] G. Plett, "High-performance battery-pack power estimation using a dynamic cell model," IEEE Transactions on Vehicular Technology, vol. 53, no. 5, pp. 1586 - 1593, Sept. 2004.

[6] K. A. Smith, C. D. Rahn, and C.-Y. Wang, "Model-based electrochemical estimation and constraint management for pulse operation of lithium ion batteries," IEEE Transactions on Control Systems Technology, vol. 18 , no. 3, pp. $654-663,2010$.

[7] R. Klein, N. A. Chaturvedi, J. Christensen, J. Ahmed, R. Findeisen, and A. Kojic, "Optimal charging strategies in lithium-ion battery," in Proceedings of the American Control Conference, San Francisco, CA, United States, 2011, pp. $382-387$.

[8] K. Thomas, J. Newman, and R. Darling, Advances in Lithium-Ion Batteries. New York, NY USA: Kluwer Academic/Plenum Publishers, 2002, ch. 12: Mathematical modeling of lithium batteries, pp. 345-392.

[9] N. A. Chaturvedi, R. Klein, J. Christensen, J. Ahmed, and A. Kojic, "Algorithms for advanced battery-management systems," IEEE Control Systems Magazine, vol. 30, no. 3, pp. 49 - 68, 2010.

[10] J. Newman. (2008) Fortran programs for the simulation of electrochemical systems. [Online]. Available: http://www.cchem.berkeley.edu/jsngrp/fortran.html

[11] J. Christensen, "Modeling diffusion-induced stress in li-ion cells with porous electrodes," Journal of the Electrochemical Society, vol. 157, no. 3, pp. A366 - A380, 2010.

[12] K. A. Smith, C. D. Rahn, and C.-Y. Wang, "Control oriented 1D electrochemical model of lithium ion battery," Energy Conversion and Management, vol. 48, no. 9, pp. 2565 - 2578, 2007. [Online]. Available: http://dx.doi.org/10.1016/j.enconman.2007.03.015

[13] V. R. Subramanian, V. Boovaragavan, V. Ramadesigan, and M. Arabandi, "Mathematical model reformulation for lithium-ion battery simulations: Galvanostatic boundary conditions," Journal of the Electrochemical Society, vol. 156, no. 4, pp. A260 - A271, 2009.

[14] J. C. Forman, S. Bashash, J. L. Stein, and H. K. Fathy, "Reduction of an electrochemistry-based li-ion battery model via quasi-linearization and pade approximation," Journal of the Electrochemical Society, vol. 158, no. 2, pp. A93 - A101, 2011.

[15] R. L. Burden and D. Faires, Numerical Analysis, Ed. 7. Brooks/Cole, 2001.

[16] S. J. Moura, N. Chaturvedi, and M. Krstic, "Adaptive PDE Observer for Battery SOC/SOH Estimation," in 2012 ASME Dynamic Systems and Control Conference, Ft. Lauderdale, FL USA, 2012.

[17] T.-K. Lee, Y. Kim, A. Stefanopoulou, and Z. Filipi, "Hybrid electric vehicle supervisory control design reflecting estimated lithium-ion battery electrochemical dynamics," in American Control Conference (ACC), July 2011, pp. $388-395$.

[18] S. J. Moura, J. L. Stein, and H. K. Fathy, "Battery-Health Conscious Power Managementin Plug-In Hybrid Electric Vehicles via Electrochemical Modeling and Stochastic Control," IEEE Transactions on Control Systems Technology, vol. PP, no. 99, p. 1, 2012. 